\title{
Prêteur en dernier ressort et solidarité de place
}

\author{
François MARINI* \\ Cerpem \\ Université Paris IX-Dauphine \\ e-mail: Francois.Marini@dauphine.fr
}

Mai 2002

\section{Introduction}

L'intervention de l'Etat dans l'économie se justifie lorsque la décentralisation des décisions conduit à une allocation sous-optimale des ressources. La vulnérabilité du système bancaire aux crises de liquidité et au risque de système est au fondement du rôle de prêteur en dernier ressort (PDR par la suite) des Banques centrales ${ }^{1}$.

La crise de liquidité a été modélisée par Diamond-Dybvig (1983) (DD par la suite). Dans leur modèle, les individus subissent un risque d'illiquidité car ils sont incertains de la période à laquelle ils désireront consommer et ils ne peuvent investir que dans une technologie irréversible. Une banque peut assurer ce risque en émettant un contrat de dépôt à vue. Comme la période à laquelle un individu désire consommer est une information privée, ce contrat d'assurance est une option: il permet à chaque individu de choisir librement la période à laquelle il retire son dépôt. En conséquence, la banque est illiquide car si chaque déposant demande un retrait anticipé, elle ne pourra pas honorer toutes ses promesses. Il s'ensuit que si chaque déposant anticipe

${ }^{*}$ Je remercie Joel Métais, François Etner et Jérôme de Boyer des Roches et les membres du séminaire CERPEM-CREFED pour les discussions que nous avons eues. Je reste seul responsable des éventuelles erreurs et imprécisions.

${ }^{1}$ Voir Freixas, Giannini, Hoggarth et Soussa (2000) pour une revue de littérature 
que tous les autres vont paniquer, ie demander un retrait anticipé non pas pour consommer immédiatement mais pour stocker et consommer plus tard, il est rationnel pour chacun de paniquer car la banque fera faillite. Cet équilibre à tache solaire sous-optimal est une crise de liquidité.

DD ont montré que la banque peut éliminer simplement ces crises en annonçant qu'elle suspendra la convertibilité lorsque les demandes totales de retraits sont égales au nombre d'individus qui ont besoin de liquidité. Ce résultat n'est cependant pas général: Engineer (1989) a démontré que si on rajoute une période dans le modèle, il n'est pas nécessairement vérifié.

DD ont également soutenu que leur modèle montre comment un organisme d'assurance des dépôts qui taxe les déposants qui ont paniqué au profit de ceux qui n'ont pas paniqué exclut les crises de liquidité. Wallace (1988) et Mc Culloch et Yu (1998) ont montré que cette politique fiscale ne peut pas être mise en oeuvre si l'assureur est soumis, comme la banque, à une contrainte de service séquentiel. La solution proposée par Marini (2001) est que l'assureur est doté d'un capital qui s'interprète du point de vue de la banque comme du capital en hors-bilan.

DD ne modélisent pas explicitement le PDR. Ils se contentent d'affirmer qu'il devrait mettre en oeuvre la même politique fiscale redistributive que celle mise en oeuvre par l'assureur des dépôts. En effet, nous pouvons lire en page 404: "Other institutions such as the discount window ("lender of last resort") may serve a similar function; however we do not model this here" et en page 417: "The Federal Reserve discount window can, as a lender of last resort, provide a similar service to deposit insurance. It would buy bank assets with (money creation) tax revenues". Clairement, cette approche se heurte à la même difficulté que celle décrite plus haut.

Dans ce papier, nous montrons simplement que dans le modèle de DD, si une banque dispose de suffisamment de richesse, elle peut intervenir comme PDR. Cette approche complète celle de Marini (2001). En effet, nous concluons de ces deux travaux que les crises de liquidité ne peuvent être éliminées que si la banque dispose de suffisamment de capital en hors-bilan. Ce résultat complète Cooper et Ross (1998) et Dowd (2000) qui montrent que si la banque a suffisamment de capital en-bilan, elle n'est pas vulnérable aux crises de liquidité.

Une autre justification du PDR réside dans les externalités négatives que la faillite d'une banque impose sur les autres banques, ou en d'autres termes la contagion des faillites bancaires. Freixas (1999) distingue la contagion 
qui résulte du changement des anticipations des déposants de celle qui est consécutive aux crédits interbancaires. En matière de contagion par les anticipations, Freixas (1999) distingue deux mécanismes: la spéculation pure et la similitude des actifs.

La contagion purement spéculative est celle modélisée par DD. Il s'agit d'un équilibre à tache solaire dans lequel les déposants d'une banque observent la faillite d'une autre banque, chacun anticipe que tous les autres vont paniquer, et alors il est rationnel pour chacun de paniquer ${ }^{2}$. Smith (1991) propose également un modèle ayant la même structure que DD et qui permet de rendre compte des ruées contagieuses durant la période du National Banking.

Le second type de contagion résulte simplement du fait que les banques investissent dans des actifs dont les rendements sont corrélés. Lorsqu'une banque fait faillite, les déposants des banques qui ont investi dans des actifs identiques sont rationnellement conduits à retirer leurs dépôts le plus vite possible.

Comme le souligne Freixas (1999), la distinction entre ces deux types de contagion est importante car dans le premier cas la contagion conduit à la liquidation de banques solvables, alors que dans le second cas elle conduit à la liquidation de banques insolvables

Du point de vue empirique, l'importance relative de ces différentes formes de contagion fait débat. Friedman et Schwartz (1963) soutiennent que durant la grande dépression des années 30 les paniques concernaient essentiellement des banques illiquides et solvables. Ils justifient leur thèse par l'absence de détérioration des fondamentaux macroéconomiques suffisamment importante pouvant justifier la soudaineté des paniques. La crise bancaire d'octobre 1930 aurait été aggravée par la faillite de la Bank of United States qui était solvable. Ce point est cependant contesté par Lucia (1985) qui soutient que la Bank of United States était probablement insolvable. Une polémique s'en

\footnotetext{
${ }^{2}$ Freixas, Parigi et Rochet (2000) généralisent le modèle de DD au cas où il existe plusieurs banques dans des localités différentes, et les déposants sont incertains du lieu où ils désireront consommer. Les déplacements des déposants entrainent des transferts interbancaires de fonds. Si une ruée se produit sur une banque A, il est optimal pour les déposants de la banque $\mathrm{B}$ qui désirent consommer en A de ne pas transférer leurs fonds, mais de les retirer de la banque $\mathrm{B}$. La ruée sur la banque $\mathrm{A}$ est alors contagieuse à la banque B. Comme les auteurs interprètent la dotation des individus comme un stock de monnaie, un PDR qui en a le monopole d'émission peut éliminer les paniques
} 
est suivie avec Friedman et Schwartz (1986). Donaldson (1992) trouve que conformément à la théorie de $\mathrm{DD}$, les paniques bancaires sont des événements imprévisibles. En identifiant des chocs économiques régionaux, Calomiris et Mason (2000) ont établi un lien étroit entre les fondamentaux et la probabilité de faillite bancaire. L'analyse de Wicker (1996) établit que les deux mécanismes de contagion ont été à l'oeuvre durant les crises de 1930-1933. Pour la période du National Banking, Wicker (2000) montre que la majorité des banques ("national banks") qui ont suspendu la convertibilité ont réouvert leurs portes peu de jours après. Il interprète ce fait comme un indice fort que ces banques étaient solvables. On ne peut toutefois pas en conclure que toutes les paniques étaient dûes à une contagion des ruées à la DD car il n'y a pas de données pour beaucoup de "state banks" et de "trust companies". Cependant, la panique de 1893 confirme l'hypothèse de contagion à la DD car la majorité des dépôts dont la convertibilité avait été suspendue était auprès des "national banks". En revanche, Carlson (2002) aboutit à des résultats ambigus. O Grada et White (2002) étudient les ruées de 1854 et 1857 sur la Emigrant Savings Industrial Bank de New York. Ils trouvent que la ruée de 1854 a été dûe à une contagion à la DD. Pour celle de 1857, la contagion liée aux asymétries d'information sur les actifs bancaires a été la cause essentielle, bien que la contagion à la DD a été également présente. Les études expérimentales de Madiès (2000) confirment également que les déposants peuvent paniquer sans que cela soit justifié par les fondamentaux.

Dans la doctrine de Bagehot, le PDR n'est concerné que par le premier type de contagion. Cette thèse est cependant critiquée par Goodhart (1988) qui soutient qu'une telle distinction est une utopie car la Banque centrale ne peut pas distinguer les banques solvables des banques insolvables.

En conséquence, une Banque centrale supporte un risque de crédit quand elle prête à une banque subissant une ruée. Elle doit alors disposer d'un capital suffisant pour assurer que la confiance en la monnaie ne sera pas remise en cause.

On retrouve ici l'interprétation donnée par de Boyer (1998) de la doctrine de Thornton: "The Bank of England consolidates the system by assuming the function of lender of last resort. It is able to hold this role for it disposes of the necessary shareholder's funds, and thus occupies a position that no other bank can dispute.(...) The importance of the Bank of England's shareholder's funds, rather than the total amount of its cash reserve, allows it to maintain its role as Central Bank" 
Goodhart et Schoenmaker (1995) soulignent les limites qu'imposait le capital de la Banque d'Angleterre dans la gestion des crises:

"Yet this latter function of crisis management, was limited in scale and scope. It was limited in scale, because the amount of central bank's shareholder's funds (the shareholders in the Bank of England being in the private sector until nationalisation in 1946), which could be applied, and possibly lost, without causing a scandal and a public outcry, was strictly limited. Hence the Bank of England, and most of other central banks in such circumstances, acted, and continue to act, in most cases where considerable sums are at risk as a primus inter pares, organising and leading a joint rescue partly of the relevant group of banks. Except in cases involving relatively small amounts, the central bank has rarely been able, or willing, to act on its own. In that respect the rescues orchestrated by the central bank, but largely financed by the other associated commercial banks, are not dissimilar to those arranged by a collectivity of commercial banks acting jointly in a Clearing House, as used to occur in the USA" (Goodhart and Schoenmaker (1995), p.541)

Nakaso (2001) montre que c'est un aspect essentiel de la crise bancaire japonaise. Le 9 décembre 1994, les deux coopératives de crédit Tokyo Kyowa et Anzen ont fait faillite. Le jugement des autorités était que ces faillites pouvaient déclencher des ruées sur d'autres institutions financières. Le plan de sauvetage comprenait la création d'une nouvelle banque, la Tokyo Kyoudou Bank (TKB), chargée d'assurer la pérennité des fonctions (en particulier celles relatives aux dépôts) des deux banques en faillite. Le capital de TKB a été souscrit à hauteur de 20 milliards de Yens par la Banque du Japon et de 20 milliards par des institutions financières privées. L'injection de capital dans TKB était basée sur l'article 25 de la loi de la Banque du Japon qui était la base légale pour la fonction de PDR. Cet article prévoyait que la Banque du Japon pouvait fournir de la liquidité, mais aussi prendre le risque de fournir du capital. La Banque a perdu $82 \%$ du capital injecté dans TKB, et la totalité des 80 milliards injectés dans Nippon Credit Bank en 1997. Nous pouvons citer Nakaso sur les conséquences de ces pertes:

"The losses were reported in the financial statement of the Bank of Japan. It was feared that this could undermine confidence in the central bank, or the national currency more broadly. For the central bank to incur losses implies, as is obvious from its balance sheet, impairment of the assets against which banknotes are issued". 
Thornton disait déjà la même chose en 1802:

"The credit, however, of even the best substitute, would be far inferior to that of the old and known Bank of England notes; for the new paper would be guaranteed by a capital probably far less ample than that of the Bank of England"

L'organisation par les autorités d'une contribution des institutions financières privées dans la constitution du capital de TKB a été le premier cas de solidarité de place au Japon (appelée approche hougachou). On comprend donc bien que pour la Banque centrale, la solidarité de place permet de limiter le risque de crédit qui est inhérent à la fonction de PDR.

L'autre cas dans lequel de PDR doit organiser une solidarité de place est celui dans lequel il n'a pas le pouvoir de créer la monnaie qu'il prête, comme le FMI par exemple. C'est alors son capital qui détermine le volume des crédits qu'il peut accorder. Si son capital est insuffisant, il faut faire appel au secteur privé.

On peut donc naturellement en suivant Fischer (1999), distinguer deux fonctions du PDR. Premièrement, il peut être le fournisseur ultime de liquidité au marché ou à des institutions individuelles. Deuxièmement, il peut être le gestionnaire de la crise sans accorder de crédits. En effet, une panique est la coordination sur un équilibre sous-optimal. Une institution ayant suffisamment d'autorité peut résoudre ce défaut de coordination, par exemple en encourageant les institutions financières vulnérables au risque systémique à accorder suffisamment de crédits à une institution financière dont la faillite serait contagieuse. La Fed a joué ce rôle dans le sauvetage de LTCM $^{3}$

Dans ce papier, nous étendons le modèle de DD au cas où il existe deux puis trois banques. Le résultat principal est que lorsque la ruée sur une banque est contagieuse aux deux autres, celles-ci font face à un problème de coordination de leur comportement. L'origine de ce problème est qu'une ruée engendre une perte sociale de bien-être car elle conduit à la liquidation anticipée d'investissements qui ne sont pas totalement réversibles. Pour éviter les paniques, les banques doivent pouvoir s'engager à être solidaires de la banque subissant la ruée initiale. Si elles endossent la perte sociale, les déposants n'ont aucune incitation à se ruer sur la première banque car celle-ci devient parfaitement liquide. Les banques solidaires doivent donc négocier la répartition de cette perte. Si le jeu de négociation est statique, il existe une

\footnotetext{
${ }^{3}$ voir Edwards (1999) pour un compte-rendu
} 
infinité d'équilibres de Nash. Typiquement, la théorie des jeux nous enseigne que dans une telle configuration un arbitre indépendant peut sélectionner un équilibre en appliquant la solution de Nash qui repose sur les principes d'efficience et d'équité. Deux interprétations de cet arbitre indépendant sont possibles. Premièrement, un banquier ayant un fort charisme au sein d'une chambre de compensation. Wicker (2000) site le cas de George Simmons Coe, Président de la chambre de compensation de New York (CCNY par la suite) durant la panique de 1873. Deuxièmement, une banque centrale dans sa fonction de gestionnaire d'une crise de liquidité contagieuse.

\section{Le prêteur en dernier ressort dans le modèle de DIAMOND - DYBVIG}

On raisonne sur trois périodes $(T=0,1,2)$. Il existe $N$ individus, avec $N$ très grand. La période à laquelle un individu désire consommer est incertaine:

$$
U\left(c_{1}, c_{2}\right)=\left\{\begin{array}{l}
U\left(c_{1}\right) \text { avec } \quad \text { probabilité } t \\
U\left(c_{2}\right) \text { avec } \text { probabilité } 1-t
\end{array}\right.
$$

où $c_{1}$ et $c_{2}$ sont les consommations aux périodes 1 et 2 respectivement. Les agents qui désirent consommer en $T=1$ sont de type 1 , et ceux qui désirent consommer en $T=2$ sont de type 2 . $N$ étant très grand, les proportions d'agents des types 1 et 2 dans l'économie sont respectivement $t$ et $1-t$. Les agents sont informés de leur type en $T=1$, et cette information est privée. A la période 0 , chaque individu est doté d'une unité de bien et il existe la technique:

$$
\begin{array}{ccc}
T=0 & T=1 & T=2 \\
& 0 & R \\
-1 & & \\
& 1 & 0
\end{array}
$$

où $R>1$ et le choix entre les profils $(0, R)$ et $(1,0)$ est effectué en $T=1$.

DD ont montré que l'équilibre lorsqu'il existe des marchés concurrentiels conduit à une allocation identique à l'autarcie dans laquelle $c_{1}^{1}=1, c_{2}^{1}=$ $0, c_{1}^{2}=0, c_{2}^{2}=R$ où $c_{T}^{k}$ est la consommation à la période $T$ d'un individu de type $\mathrm{k}$, et l'utilité attendue en $T=0$ est $t U(1)+(1-t) U(R)$. 
Supposons à présent que les individus forment une banque, c'est à dire qu'ils mettent en commun leurs dotations, les investissent dans la technologie, et obtiennent en contrepartie un contrat de dépôt à vue qui spécifie le nombre d'unités du bien qu'ils peuvent retirer en $T=1$ ou en $T=2$, notés respectivement $r_{1}$ et $r_{2}$. Afin de financer les retraits en $T=1$, la banque liquide $L$ unités par tête en $T=1$ et reçoit donc $R(1-L)$ unités par tête en $T=2$. La banque émet le contrat qui résout:

$$
\text { sous } \begin{gathered}
t r_{1}=L \\
(1-t) r_{2}=R(1-L) \\
r_{2}>r_{1}
\end{gathered}
$$

avec $r_{1}=c_{1}^{1}$ et $r_{2}=c_{2}^{2}$. On remarque qu'en plus de sa contrainte de budget, la banque fait face à la contrainte incitative $r_{2}>r_{1}$, c'est à dire qu'aucun agent de type 2 ne doit avoir intérêt à retirer en $T=1$. Si le coefficient d'aversion relative à l'égard du risque est supérieur à 1 , c'est à dire si $-x U^{\prime \prime}(x) / U^{\prime}(x)>1$, cela signifie que $x U^{\prime}(x)$ est une fonction décroissante de $x$, d'où $R U^{\prime}(R)<U^{\prime}(1)$. Or à l'équilibre $U^{\prime}\left(r_{1}\right)=R U^{\prime}\left(r_{2}\right)$. Il s'ensuit donc qu'à l'équilibre $r_{1}>1$ et $r_{2}<R$. En outre, $R>1$ implique que $U^{\prime}\left(r_{1}\right)>U^{\prime}\left(r_{2}\right)$ d'où $r_{2}>r_{1}$. La banque assure les déposants contre le risque d'être de type 1 en se mettant en position d'illiquidité.

Le fait que les types des agents sont des informations privées implique que la banque ne peut assurer les déposants contre le risque d'être de type 1 qu'en les laissant choisir librement la période à laquelle ils retirent et en leur procurant le rendement promis. Mais si beaucoup de déposants désirent retirer en $T=1$, tous ne pourront pas être remboursés car la banque est illiquide. Seuls les premiers s'étant présentés à la banque le seront. Les déposants font donc face à une contrainte de service séquentiel qui s'écrit formellement:

$$
\widetilde{r}_{1}=\left\{\begin{array}{lll}
r_{1} & \text { avec } & \text { la probabilité } \Gamma=\min (1,1 / w) \\
0 & \text { avec } & \text { la probabilité } 1-\Gamma
\end{array}\right.
$$

où $w$ sont les demandes totales de retraits et 1 est la valeur liquidative de la banque en $T=1$. Si $w<1$ chaque déposant désirant retirer en $T=1$ obtient avec certitude le rendement $r_{1}$, alors que si $w>1$ chaque déposant désirant 
retirer en $T=1$ obtient $r_{1}$ avec la probabilité $1 / w$ et 0 avec la probabilité $1-(1 / w)$.

Si l'on note $\theta_{i j}$ la proportion des agents de type i qui déclarent être de type j (nous reprenons dans ce qui suit Marini (1992)), alors:

$$
w=\left(t \theta_{11}+(1-t) \theta_{21}\right) r_{1}=f r_{1}
$$

et les déposants qui retirent en $T=2$ obtiennent:

$$
\widetilde{r}_{2}=\max (0 ; R(1-w) /(1-f))
$$

L'illiquidité de la banque implique qu'en $T=1$ les déposants sont dans une situation d'intéraction stratégique car le rendement que chaque déposant perçoit dépend des types que les autres déposants ont déclarés, ou en d'autres termes $r_{1}$ et $r_{2}$ sont fonctions des $\theta_{i j}$. Si l'on raisonne en stratégies pures, le jeu de révélation des types auquel jouent les déposants possède deux équilibres de Nash qui sont l'équilibre de vérité dans lequel chacun déclare son vrai type (personne ne ment), et l'équilibre de panique dans lequel tous les déposants déclarent être de type 1 et tentent de retirer en $T=1$ (les agents de type 2 mentent).

L'équilibre de vérité correspond à $\theta_{11}=1, \theta_{12}=0, \theta_{21}=0, \theta_{22}=1$; car $\theta_{11}=1$ et $\theta_{21}=0$ impliquent que $w=t r_{1}=L$ qui est nécessairement inférieur à 1 à l'équilibre, d'où il s'ensuit que $\Gamma=1$ et donc chaque déposant maximise son utilité attendue en déclarant son vrai type. L'équilibre de panique correspond à $\theta_{11}=1, \theta_{12}=0, \theta_{21}=1, \theta_{22}=0$; car $\theta_{11}=1$ et $\theta_{21}=1$ impliquent que $w=r_{1}>1$, d'où $r_{2}=0$ et donc chaque déposant de type 2 maximise son utilité attendue en paniquant, c'est à dire en déclarant être de type 1, car chacun anticipe que tous les autres vont paniquer. La panique est un équilibre à tache solaire, celle-ci pouvant être par exemple la crainte d'une invasion étrangère, ou une ruée sur une autre banque. La contagion interbancaire des ruées est donc un phénomène psychologique lié au fait que les déposants d'une banque coordonnent leurs anticipations sur celles des déposants d'une autre banque. C'est une contagion de l'anticipation de panique.

Lorsqu'il n'y a pas d'incertitude sur $t$, la suspension de la convertibilité suffit à exclure les paniques et ne réduit pas le bien-être social. Le PDR n'est donc pas nécessaire. Cependant, Engineer (1989) a démontré que la suspension de la convertibilité ne permet pas toujours d'exclure les paniques dans un 
modèle à quatre périodes. Cela provient du fait que l'anticipation d'une ruée dans le futur (en $T=2$ ) peut déclencher une ruée dans la période courante (en $T=1$ ). Dans ce cas, la menace d'une suspension de la convertibilité en $T=1$ ne permet pas de dissuader les déposants de paniquer.

L'équilibre de panique peut être éliminé par l'intervention d'un PDR. Supposons en effet que la Banque centrale ne s'intéresse qu'à la maximisation du bien-être social et dispose d'une dotation $r_{1}(1-t)$. Elle annonce qu'elle accorde une ligne de crédit de $r_{1}(1-t)$ que la banque tire à partir de $f=t$. Comme spécifié dans le contrat de dépôt à vue, la banque liquide en $T=1$ $L=t r_{1}$. S'il y a une panique, elle emprunte $(1-t) r_{1}$ à la Banque centrale, ce qui lui permet de rembourser tous les déposants au rendement promis $r_{1}$ et par conséquent la banque est sauvée. En $T=2$, elle dispose de $R\left(1-t r_{1}\right)$, ce qui lui permet de rembourser la Banque centrale à un taux d'intérêt positif car $R\left(1-t r_{1}\right)>r_{1}(1-t)$. Cette inégalité est nécessairement vérifiée car la banque a émis un contrat de dépôt à vue vérifiant $r_{2}>r_{1}$. Ce crédit garantit donc à la fois la pérennité de la banque en assurant que la totalité des déposants est remboursée au rendement $r_{1}$, et celle du PDR car la banque est illiquide et solvable.

On remarque que le rendement du crédit accordé par le $\mathrm{PDR}, R^{p}=$ $R\left(1-t r_{1}\right) / r_{1}(1-t)$, est inférieur à $r_{2}$ puisque $r_{1}>1$. Il est donc également inférieur à $R$ puisque $r_{2}<R$ à l'équilibre. Ceci provient du fait que la crise de liquidité conduit à une liquidation des investissements supérieure à ce qui est socialement optimal. Une panique bancaire a donc un coût en terme de bien-être social qui est supporté par le PDR. Si le rendement demandé par le PDR était supérieur à $R\left(1-t r_{1}\right) / r_{1}(1-t)$, il mettrait la banque en situation d'insolvabilité et ainsi ne pourrait pas éliminer l'équilibre de panique.

Ce résultat nuance le principe de Bagehot selon lequel le PDR devrait s'engager à prêter à un taux d'intérêt pénalisant. Certes, $R\left(1-t r_{1}\right) / r_{1}(1-t)$ est forcément supérieur à 1 , ie au rendement du stockage ou de la monnaie. Mais si le PDR veut assurer la pérennité de la banque (et nous avons vu que Nakaso (2001) montre que cela a toujours été un objectif essentiel de la Banque du Japon, et qui a été déterminant dans les plans de sauvetage), il doit supporter la perte sociale engendrée par la panique. C'est donc le PDR, et non pas la banque, qui est pénalisé. Humphrey $(1975,1989)$ analyse les fondements du principe de Bagehot. Le modèle de DD ne remet pas en cause ces fondements. Il montre simplement une difficulté à laquelle le principe de Bagehot pourrait se heurter. 
Deux interprétations sont possibles pour la dotation $r_{1}(1-t)$. Premièrement, si le PDR a le pouvoir de création monétaire, $r_{1}(1-t)$ est la quantité de monnaie qu'il prête. Le modèle de DD est alors interprété comme un modèle monétaire, ie que les dotations des agents sont de la monnaie. C'est l'interprétation de Freixas, Parigi et Rochet (2000) dans leur note 6. On peut alors considérer que le $\mathrm{PDR}$ rachète $\left(1-t r_{1}\right)$ unités de capital de la banque en créant $r_{1}(1-t)$ unités de monnaie. On retrouve alors la définition que donnent DD du PDR et que nous avons cité dans l'introduction. Deuxièmement, si le PDR n'a pas le pouvoir de création monétaire, $r_{1}(1-t)$ est son capital. C'est le cas d'une Banque centrale qui doit prêter en une monnaie qu'elle ne crée pas, ou le cas du Fonds Monétaire International. Le capital peut alors être insuffisant pour éliminer l'équilibre de panique. C'est la thèse développée par Sachs (1998) sur les crises des marchés émergents. Le FMI n'a pas joué son rôle de PDR car les prêts qu'il a accordés étaient insuffisants au regard des fuites de capitaux potentielles.

\section{Faillite du marché monétaire et solidarité de place dans un modèle à deux banques}

Nous supposons à présent que l'économie est composée de deux ensembles infinis d'individus ayant le même cardinal (deux continuum sur $[0 ; 1]$ ). L'ensemble $X$ comprend les individus qui ont un coefficient d'aversion relative à l'égard du risque $\alpha$, et l'ensemble $Y$ comprend les individus qui ont un coefficient d'aversion relative à l'égard du risque $\beta$. Montrons d'abord que les deux ensembles ne vont pas constituer une seule coalition, mais deux coalitions, l'une composée des individus appartenant à l'ensemble $X$ et l'autre composée des individus appartenant à l'ensemble $Y$.

Supposons qu'une proportion $t$ des individus de $X$ est de type 1 , et une proportion $t^{\prime}>t$ des individus de $Y$ est de type 1. Si les deux ensembles se constituent en une seule coalition, alors une proportion $\left(t+t^{\prime}\right) / 2$ des individus de l'unique coalition est de type 1 . Nous pouvons ainsi représenter graphiquement les contraintes budgétaires de ces trois coalitions qui passent nécessairement par le point $(1, R)$.

Les contrats de dépôt à vue étant situés sur les segments allant du point $\mathrm{A}$ à la droite à 45 degrés (puisque $1<r_{1}<r_{2}<R$ ), il apparait clairement sur le 


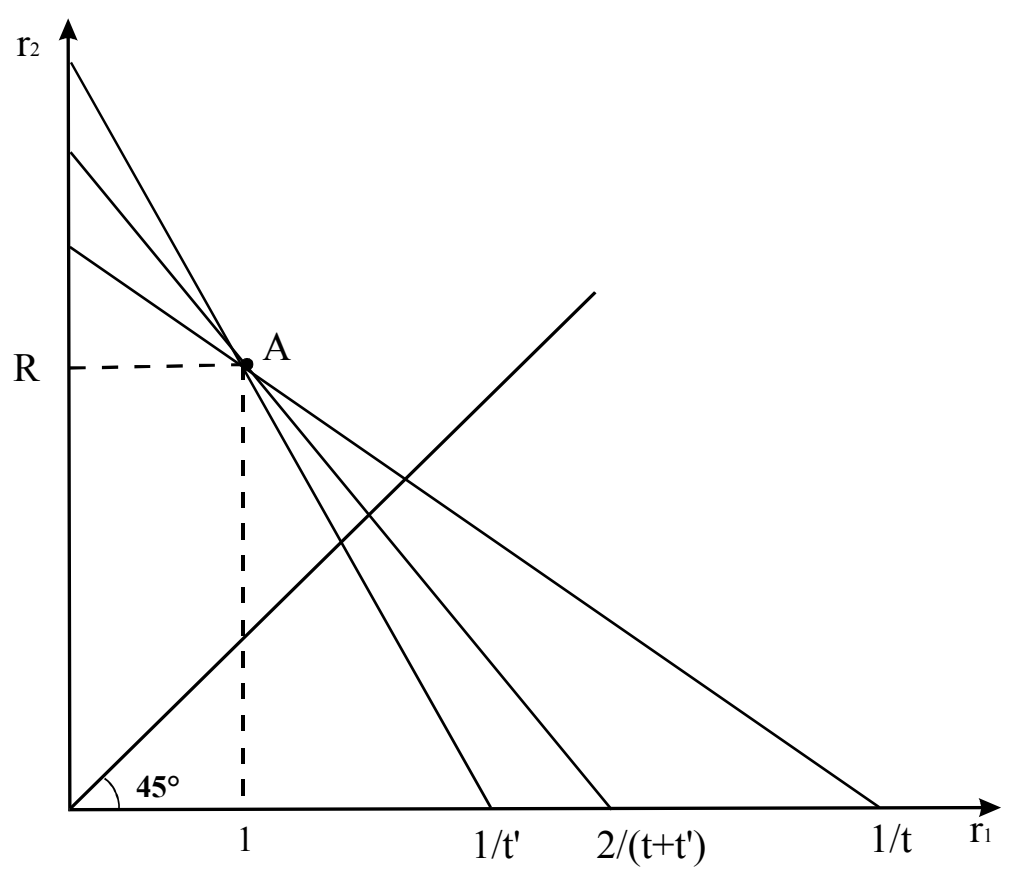

Figure 1:

graphique 1 que l'ensemble $Y$ a intérêt à former une coalition avec l'ensemble $X$, mais l'ensemble $X$ n'a pas intérêt à former une coalition avec l'ensemble $Y$. En outre, l'hypothèse $t=t^{\prime}$ est naturelle car on ne voit pas pourquoi la probabilité de subir un choc de liquidité en $T=1$ dépendrait du coefficient d'aversion relative à l'égard du risque. Ainsi, comme nous supposons que la proportion d'individus de type 1 est la même dans les deux ensembles $X$ et $Y$, ceux-ci ne peuvent pas accroitre l'ensemble des consommations possibles en formant une seule coalition. Une hypothèse naturelle est qu'une coalition ne se forme que si elle augmente le bien-être social de ses membres. Par conséquent, il n'y a pas de perte de généralité à supposer que le système bancaire est composé des deux banques $X$ et $Y$ qui émettent les contrats $\left(r_{1}^{X}, r_{2}^{X}\right)$ et $\left(r_{1}^{Y}, r_{2}^{Y}\right)$ plutôt qu'une seule banque qui proposerait ces deux 
contrats. Notre analyse n'est pas modifiée si l'on retient cette deuxième formulation. Il suffit de remplacer "banque $X, Y$ ou $Z$ " par "déposants $X, Y$ ou Z".

Nous supposons en outre que si une tache solaire apparait au début de la première période, une ruée se déclenche sur la banque $X$ qui est contagieuse à la banque $Y$. Le mécanisme de la contagion est donc celui de la contagion des anticipations de panique décrit plus haut.

Avec la fonction d'utilité élémentaite $U(c)=\frac{(c+\delta)^{1-\alpha}}{1-\alpha}$, où $\delta$ est un paramètre positif. Les rendements du contrat de dépôt sont:

$$
\begin{gathered}
r_{1}=\frac{1-(1-t) \delta\left(R^{(1-\alpha) / \alpha}-(1 / R)\right)}{t+(1-t) R^{(1-\alpha) / \alpha}} \\
r_{2}=R^{1 / \alpha}\left(r_{1}+\delta\right)-\delta
\end{gathered}
$$

Notons $E C_{X}$ l'équivalent certain de la loterie qui rapporte $r_{1}^{X}$ avec la probabilité $1 / r_{1}^{X}$ ou 0 avec la probabilité $1-1 / r_{1}^{X}$, et $E C_{Y}$ l'équivalent certain de la loterie qui rapporte $r_{1}^{Y}$ avec la probabilité $1 / r_{1}^{Y}$ ou 0 avec la probabilité $1-1 / r_{1}^{Y}$. Nous avons:

$$
E C_{X}=\left\{\left(1 / r_{1}^{X}\right)\left[\left(r_{1}^{X}+\delta^{X}\right)^{1-\alpha}-\left(\delta^{X}\right)^{1-\alpha}\right]\right\}^{\frac{1}{1-\alpha}}-\delta^{X}
$$

et $E C_{Y}$ est défini de façon identique avec $r_{1}^{Y}$ à la place de $r_{1}^{X}$ et $\delta^{Y}$ à la place de $\delta^{X}$.

Si la banque $X$ liquide 1 en $T=1$ et emprunte $r_{1}^{X}-1$, elle ne pourra pas rembourser son emprunt. Si elle liquide $L^{X}=t r_{1}^{X}$, elle doit emprunter $r_{1}^{X}-L^{X}=(1-t) r_{1}^{X}$. Elle pourra rembourser en $T=2 R\left(1-L^{X}\right)$. Le rendement de ce prêt est $R^{P}=R\left(1-L^{X}\right) /\left(r_{1}^{X}-L^{X}\right)$ qui est inférieur à $R$. Cela s'explique par le fait que la quantité d'actif liquidée en $T=1$ est supérieure à ce qui est socialement optimal. 
Si la banque $\mathrm{Y}$ fait supporter la perte aux agents de type 1, le prêt par tête maximum $P_{1}^{\prime}$ qu'elle peut accorder à la banque $\mathrm{X}$ est donné par $L^{Y}-P_{1}^{\prime}=t E C_{Y}$, soit $P_{1}^{\prime}=t\left(r_{1}^{Y}-E C_{Y}\right)$. Il y aura faillite du marché monétaire si:

$$
t\left(r_{1}^{Y}-E C_{Y}\right)<(1-t) r_{1}^{X}
$$

où $r_{1}^{Y}-E C_{Y}$ est le rendement maximum auquel peut renoncer un agent de type 1 sans qu'il soit incité à paniquer.

Si la perte est supportée par les agents de type 2 , le prêt maximum $P_{2}^{\prime}$ que peut accorder la banque Y est donné par:

$$
\frac{1}{1-t}\left[P_{2}^{\prime} R^{P}+\left(1-L^{Y}-P_{2}^{\prime}\right) R\right]=r_{1}^{Y}
$$

d'où on obtient $P_{2}^{\prime}=(1-t)\left(r_{2}^{Y}-r_{1}^{Y}\right) /\left(R-R^{P}\right)$. Il y aura donc faillite du marché monétaire si $P_{2}^{\prime}<r_{1}^{X}-L^{X}$, soit $\left(r_{2}^{Y}-r_{1}^{Y}\right)<\left(R-R^{P}\right) r_{1}^{X}$. Le terme $\left(r_{2}^{Y}-r_{1}^{Y}\right)$ est le rendement maximum auquel peut renoncer un agent de type 2 sans qu'il soit incité à paniquer. Le terme $R-R^{P}$ est la perte de rendement liée à la liquidation d'actif plus importante que ce qui est socialement optimal.

On peut également considérer le cas dans lequel les agents des types 1 et 2 de la banque $Y$ ne peuvent pas isolément sauver la banque $X$, mais ensemble ils le peuvent:

$$
P_{1}^{\prime}<(1-t) r_{1}^{X} ; P_{2}^{\prime}<(1-t) r_{1}^{X} ; P_{1}^{\prime}+P_{2}^{\prime} \geq(1-t) r_{1}^{X}
$$

Ils doivent donc négocier leurs prêts à la banque $X$. Nous supposons que les déposants de la banque $Y$ jouent simultanément. Cette hypothèse est réaliste car en situation de panique potentielle, les banques doivent réagir rapidement, ou en d'autres termes la négociation doit aboutir rapidement. Une bonne approximation est donc que les déposants jouent simultanément car ils n'ont pas le temps de s'engager dans un processus long d'offres et de contre-offres. Nous sommes donc dans la même configuration que dans le jeu de négociation de Nash, sauf qu'au lieu de se répartir un surplus, les joueurs 
se répartissent une perte. ${ }^{4}$ Nous traitons les agents d'un certain type de façon symétrique. L'ensemble des accords possibles est:

$$
A=\left\{\left(P_{1} ; P_{2}\right) \in R^{2}: P_{1} \leq P_{1}^{\prime} ; P_{2} \leq P_{2}^{\prime} ; P_{1}+P_{2} \geq(1-t) r_{1}^{X}\right\} \cup\{(0,0)\}
$$

L'ensemble des équilibres de Nash symétriques est:

$$
N=\left\{\left(P_{1} ; P_{2}\right) \in R^{2}: P_{1} \leq P_{1}^{\prime} ; P_{2} \leq P_{2}^{\prime} ; P_{1}+P_{2}=(1-t) r_{1}^{X}\right\} \cup\{(0,0)\}
$$

En effet, si chaque agent de type 1 offre $P_{1}$, la meilleure réponse de chaque agent de type 2 est $P_{2}=(1-t) r_{1}^{X}-P_{1}$. Prêter plus ou moins réduirait l'utilité des agents de type 2. Symétriquement, si chaque agent de type 2 offre $P_{2}$, la meilleure réponse de chaque agent de type 1 est $P_{1}=(1-t) r_{1}^{X}-P_{2}$. En outre, comme aucun type d'agent ne peut sauver seul la banque $X$, si les agents d'un certain type offrent 0 , la meilleure réponse de l'autre type est 0 . Cet équilibre de Nash est sous-optimal au sens de Pareto.

Comme il existe une infinité d'équilibres de Nash, les déposants font face à un problème de coordination. Trois possibilités peuvent alors être envisagées. Premièrement, les propriétés d'efficience et d'équité font de la solution de Nash un équilibre focal sur lequel les joueurs se coordonnent même s'il n'y a pas d'arbitre. Deuxièmement, un arbitre dicte aux joueurs les stratégies d'équilibre qu'ils doivent jouer et qui conduisent à la solution de Nash. Troisièmement, l'équilibre focal est déterminé par une négociation avant que les joueurs jouent (voir Myerson (1991) et Thomson (1994)). Nous interprétons l'arbitre indépendant comme le PDR dans sa fonction de gestionnaire d'une crise de liquidité.

La fonction d'utilité étant strictement concave et bornée, l'ensemble des utilités possibles est compact et convexe. Soient $r_{1 e}^{Y}$ et $r_{2 e}^{Y}$ les rendements effectifs que la banque $Y$ verse aux agents de type 1 et 2 respectivement lorsqu'elle est solidaire de la banque $X$. Nous avons $\operatorname{tr}_{1 e}^{Y}=L_{e}^{Y} \leq L^{Y}$ et $(1-t) r_{2 e}^{Y}=R\left(1-L_{e}^{Y}-(1-t) r_{1}^{X}\right)+R^{P}(1-t) r_{1}^{X}$. Les prêts sont $P_{1}=$ $\left(L^{Y}-L_{e}^{Y}\right) / t$ et $P_{2}=(1-t) r_{1}^{X}-P_{1}$. L'arbitre résout:

$$
\underset{L_{e}^{Y}}{\operatorname{Max}}\left[U_{Y}\left(r_{1 e}^{Y}\right)-U_{Y}\left(E C_{Y}\right)\right]^{N t}\left[U_{Y}\left(r_{2 e}^{Y}\right)-U_{Y}\left(E C_{Y}\right)\right]^{N(1-t)}
$$

\footnotetext{
${ }^{4}$ voir par exemple Van Damme (1991) et Osborne et Rubinstein (1991)
} 
sous $U_{Y}\left(r_{1 e}^{Y}\right) \geq U_{Y}\left(E C_{Y}\right)$

$U_{Y}\left(r_{2 e}^{Y}\right) \geq U_{Y}\left(E C_{Y}\right)$

$P_{1}+P_{2}=(1-t) r_{1}^{X}$

où $\left(U_{Y}\left(E C_{Y}\right) ; U_{Y}\left(E C_{Y}\right)\right)$ est le point de désaccord.

La solution de Nash est donnée par:

$$
\frac{U_{Y}^{\prime}\left(r_{1 e}^{Y}\right)}{U_{Y}\left(r_{1 e}^{Y}\right)-U_{Y}\left(E C_{Y}\right)}=\frac{R U_{Y}^{\prime}\left(r_{2 e}^{Y}\right)}{U_{Y}\left(r_{2 e}^{Y}\right)-U_{Y}\left(E C_{Y}\right)}
$$

Une autre façon d'organiser la solidarité de place consiste pour la banque $Y$ à verser $r_{1}^{X}-1$ à la banque $X$. Si la perte est subie par les agents de type 1, la banque $\mathrm{Y}$ a intérêt à être solidaire de la banque $\mathrm{X}$ si $r_{1}^{Y}-r_{1}^{X}+$ $1>E C_{Y}$. Si cette condition est vérifiée, la banque $\mathrm{X}$ devient parfaitement liquide et n'est donc plus vulnérable aux paniques. Ainsi, la solidarité de la banque $Y$ est parfaitement crédible, et il n'y aura donc pas de panique sur la banque $X$ même en l'absence de PDR. La solidarité de place ne sera donc pas effectivement mise en oeuvre, d'où les agents de type 1 ne supporteront pas effectivement le coût de la solidarité. De même, dans le cas d'une contagion de la banque $Y$ vers la banque $X$, la banque $X$ est solidaire de la banque $Y$ si $r_{1}^{X}-r_{1}^{Y}+1>E C_{X}$.

Si la perte est subie par les agents de type 2, la banque $Y$ liquide en $T=1$ $t r_{1}^{Y}+r_{1}^{X}-1$, d'où le rendement des agents de type 2 est $R\left(2-r_{1}^{X}-t r_{1}^{Y}\right) /(1-t)$. Si ce rendement est supérieur à $r_{1}^{Y}$, les agents de type 2 de la banque $Y$ ont intérêt à être solidaires de la banque $X$ et ils ne paniquent pas. Par un raisonnement identique, les agents de type 2 de la banque $X$ ont intérêt à être solidaires de la banque $Y$ si $R\left(2-r_{1}^{Y}-t r_{1}^{X}\right) /(1-t)>r_{1}^{X}$.

On peut également considérer le cas où les agents des type 1 et 2 de la banque $Y$ contribuent ensemble au renflouement de la banque $X$. Notons $F_{1}^{Y}$ la contribution d'un agent de type 1 et $F_{2}^{Y}$ celle d'un agent de type 2.

L'ensemble des accords possibles est:

$$
\begin{aligned}
A=\{ & \left(F_{1}^{Y} ; F_{2}^{Y}\right) \in R^{2}: F_{1}^{Y} \leq r_{1}^{Y}-E C_{Y} ; F_{2}^{Y} \leq\left(r_{2}^{Y}-E C_{Y}\right) / R ; \\
& \left.t F_{1}^{Y}+(1-t) F_{2}^{Y} \geq r_{1}^{X}-1\right\}
\end{aligned}
$$

L'ensemble des équilibres de Nash symétriques est:

$$
N=\left\{\left(F_{1}^{Y} ; F_{2}^{Y}\right) \in R^{2}: F_{1}^{Y} \leq r_{1}^{Y}-E C_{Y} ; F_{2}^{Y} \leq\left(r_{2}^{Y}-E C_{Y}\right) / R ;\right.
$$




$$
\left.t F_{1}^{Y}+(1-t) F_{2}^{Y}=r_{1}^{X}-1\right\}
$$

L'arbitre résout:

$$
\begin{array}{cc}
\operatorname{Max}_{e}^{Y}\left[U_{Y}\left(r_{1}^{Y}-F_{1}^{Y}\right)-U_{Y}\left(E C_{Y}\right)\right]^{N t}\left[U_{Y}\left(r_{2}^{Y}-R F_{2}^{Y}\right)-U_{Y}\left(E C_{Y}\right)\right]^{N(1-t)} \\
\text { sous } \quad U_{Y}\left(r_{1}^{Y}-F_{1}^{Y}\right) \geq U_{Y}\left(E C_{Y}\right) \\
U_{Y}\left(r_{2}^{Y}-R F_{2}^{Y}\right) \geq U_{Y}\left(E C_{Y}\right) \\
t F_{1}^{Y}+(1-t) F_{2}^{Y}=r_{1}^{X}-1
\end{array}
$$

Le raisonnement est symétrique pour le sauvetage de la banque $Y$ par la banque $X$.

\section{Faillite du marché monétaire et solidarité de place dans un modèle à trois banques}

Nous supposons à présent que le système bancaire comprend trois banques $X$, $Y$ et $Z$ qui émettent respectivement les contrats $\left(r_{1}^{X}, r_{2}^{X}\right),\left(r_{1}^{Y}, r_{2}^{Y}\right)$ et $\left(r_{1}^{Z}, r_{2}^{Z}\right)$. Nous considérons le cas d'une ruée sur la banque $X$ contagieuse aux banques $Y$ et $Z$.

Par un raisonnement identique à celui de la section 3 , lorsque la perte est supportée par les agents de type 1, il y a faillite du marché monétaire si:

$$
t\left(r_{1}^{Y}-E C_{Y}\right)+t\left(r_{1}^{Z}-E C_{Z}\right)<(1-t) r_{1}^{X}
$$

Lorsque $t\left(r_{1}^{Y}-E C_{Y}\right)+t\left(r_{1}^{Z}-E C_{Z}\right)>(1-t) r_{1}^{X}, t\left(r_{1}^{Y}-E C_{Y}\right)<(1-t) r_{1}^{X}$ et $t\left(r_{1}^{Z}-E C_{Z}\right)<(1-t) r_{1}^{X}$, aucune des banques $Y$ et $Z$ ne peut seule prêter suffisamment à la banque $X$. Elles doivent prêter toutes les deux pour éviter la contagion. Les banques $Y$ et $Z$ doivent donc négocier leurs contributions propres au sauvetage de la banque $X$. Il s'agit d'un jeu à somme nulle où ce qui est versé par l'une ne l'est pas par l'autre. L'ensemble des accords possibles est:

$$
\begin{aligned}
A=\{ & \left(P_{1}^{Y} ; P_{1}^{Z}\right) \in R^{2}: P_{1}^{Y}+P_{1}^{Z} \geq(1-t) r_{1}^{X} ; P_{1}^{Y} \leq L^{Y}-t E C_{Y} \\
& \left.P^{Z} \leq L^{Z}-t E C_{Z}\right\} \cup\{(0 ; 0)\}
\end{aligned}
$$


L'ensemble des équilibres de Nash est:

$$
\begin{aligned}
N=\{ & \left(P_{1}^{Y} ; P_{1}^{Z}\right) \in R^{2}: P_{1}^{Y}+P_{1}^{Z}=(1-t) r_{1}^{X} ; P_{1}^{Y} \leq L^{Y}-t E C_{Y} \\
& \left.P_{1}^{Z} \leq L^{Z}-t E C_{Z}\right\} \cup\{(0 ; 0)\}
\end{aligned}
$$

L'arbitre résout:

$$
\begin{array}{cl}
\text { Max } & {\left[U_{Y}\left(L^{Y}-P_{1}^{Y}\right)-U_{Y}\left(E C_{Y}\right)\right]\left[U_{Z}\left(L^{Z}-P_{1}^{Z}\right)-U_{Z}\left(E C_{Z}\right)\right]} \\
P_{1}^{Y} ; P^{Z} & U_{Y}\left(L^{Y}-P_{1}^{Y}\right) \geq U_{Y}\left(E C_{Y}\right) \\
\text { sous } & U_{Z}\left(L^{Z}-P_{1}^{Z}\right) \geq U_{Z}\left(E C_{Z}\right) \\
& P_{1}^{Y}+P_{1}^{Z}=(1-t) r_{1}^{X}
\end{array}
$$

La solution de Nash est donnée par:

$$
\frac{U_{Y}^{\prime}\left(L^{Y}-P_{1}^{Y}\right)}{U_{Y}\left(L^{Y}-P_{1}^{Y}\right)-U_{Y}\left(E C_{Y}\right)}=\frac{U_{Z}^{\prime}\left(L^{Z}+P_{1}^{Y}-(1-t) r_{1}^{X}\right)}{U_{Z}\left(L^{Z}-P_{1}^{Z}\right)-U_{Z}\left(E C_{Z}\right)}
$$

Lorsque la perte est supportée par les agents de type 2, le marché monétaire ne permet pas d'éviter la contagion si:

$$
\left(r_{2}^{Y}-r_{1}^{Y}\right)+\left(r_{2}^{Z}-r_{1}^{Z}\right)<\left(R-R^{P}\right) r_{1}^{X}
$$

Lorsque $\left(r_{2}^{Y}-r_{1}^{Y}\right)+\left(r_{2}^{Z}-r_{1}^{Z}\right)>\left(R-R^{P}\right) r_{1}^{X},\left(r_{2}^{Y}-r_{1}^{Y}\right)<\left(R-R^{P}\right) r_{1}^{X}$ et $\left(r_{2}^{Z}-r_{1}^{Z}\right)<\left(R-R^{P}\right) r_{1}^{X}$, l'ensemble des accords possibles est:

$$
\begin{aligned}
A=\{ & \left(P_{2}^{Y} ; P_{2}^{Z}\right) \in R^{2}: P_{2}^{Y}+P_{2}^{Z} \geq(1-t) r_{1}^{X} \\
& P_{2}^{Y} \leq\left(1 /\left(R-R^{P}\right)\right)\left[\left(1-L^{Y}\right) R-(1-t) r_{1}^{Y}\right] ; \\
& \left.P_{2}^{Z} \leq\left(1 /\left(R-R^{P}\right)\right)\left[\left(1-L^{Z}\right) R-(1-t) r_{1}^{Z}\right]\right\} \cup\{(0 ; 0)\}
\end{aligned}
$$

L'ensemble des équilibres de Nash est:

$$
\begin{aligned}
N=\{ & \left(P_{2}^{Y} ; P_{2}^{Z}\right) \in R^{2}: P_{2}^{Y}+P_{2}^{Z}=(1-t) r_{1}^{X} \\
& P_{2}^{Y} \leq\left(1 /\left(R-R^{P}\right)\right)\left[\left(1-L^{Y}\right) R-(1-t) r_{1}^{Y}\right] \\
& \left.P_{2}^{Z} \leq\left(1 /\left(R-R^{P}\right)\right)\left[\left(1-L^{Z}\right) R-(1-t) r_{1}^{Z}\right]\right\} \cup\{(0 ; 0)\}
\end{aligned}
$$

L'arbitre résout: 


$$
\begin{array}{cl}
\operatorname{Max}_{2}^{Y} ; P_{2}^{Z} & {\left[U_{Y}\left(r_{2}^{Y}\left(P_{2}^{Y}\right)\right)-U_{Y}\left(E C_{Y}\right)\right]\left[U_{Z}\left(r_{2}^{Z}\left(P_{2}^{Z}\right)\right)-U_{Z}\left(E C_{Z}\right)\right]} \\
\text { sous } & U_{Y}\left(r_{2}^{Y}\left(P_{2}^{Y}\right)\right) \geq U_{Y}\left(E C_{Y}\right) \\
& U_{Z}\left(r_{2}^{Z}\left(P_{2}^{Z}\right)\right) \geq U_{Z}\left(E C_{Z}\right) \\
& P_{2}^{Y}+P_{2}^{Z}=(1-t) r_{1}^{X}
\end{array}
$$

La solution de Nash est donnée par:

$$
\begin{gathered}
\frac{U_{Y}^{\prime}\left(r_{2}^{Y}\left(P_{2}^{Y}\right)\right)}{U_{Y}\left(r_{2}^{Y}\left(P_{2}^{Y}\right)-U_{Y}\left(E C_{Y}\right)\right.}=\frac{U_{Z}^{\prime}\left(r_{2}^{Z}\left(P_{2}^{Y}\right)\right)}{U_{Z}\left(r_{2}^{Z}\left(P_{2}^{Y}\right)\right)-U_{Z}\left(E C_{Z}\right)} \\
\text { où } r_{2}^{Z}\left(P_{2}^{Y}\right)=\frac{\left[(1-t) r_{1}^{X}-P_{1}^{Y}\right]\left(R^{P}-R\right)+\left(1-L^{Z}\right) R}{1-t}
\end{gathered}
$$

On peut également considérer le cas où il y a faillite du marché monétaire lorsque la perte est supportée par les agents d'un seul type, mais les deux types d'agents peuvent être solidaires de la banque $X$ :

$$
\begin{gathered}
t\left(r_{1}^{Y}-E C_{Y}\right)+t\left(r_{1}^{Z}-E C_{Z}\right)<(1-t) r_{1}^{X} \\
\frac{(1-t)\left[\left(r_{2}^{Y}-r_{1}^{Y}\right)+\left(r_{2}^{Z}-r_{1}^{Z}\right)\right]}{R-R^{P}}<(1-t) r_{1}^{X} \\
t\left(r_{1}^{Y}-E C_{Y}\right)+t\left(r_{1}^{Z}-E C_{Z}\right)+\frac{(1-t)\left[\left(r_{2}^{Y}-r_{1}^{Y}\right)+\left(r_{2}^{Z}-r_{1}^{Z}\right)\right]}{R-R^{P}} \geq(1-t) r_{1}^{X}
\end{gathered}
$$

Il est trivial de montrer que comme précédemment le jeu de négociation de Nash a une infinité d'équilibres, et que ce problème de coordination peut être résolu par un arbitre indépendant qui applique la solution de Nash.

Lorsque la solidarité de place consiste pour les banques $Y$ et $Z$ à verser $r_{1}^{X}-1$ à la banque $X$, elles doivent négocier leurs contributions. Notons $F_{1}^{Y}$ la contribution des agents de type 1 de la banque $Y$ dans le financement du 
sauvetage de la banque $X$, et $F_{1}^{Z}$ la contribution des agents de type 1 de la banque $Z$. Les conditions suffisantes de la solidarité sont la contrainte de ressource $F_{1}^{Y}+F_{1}^{Z} \geq r_{1}^{X}-1$ et les contraintes de participation $F_{1}^{Y} \leq r_{1}^{Y}-E C_{Y}$ et $F_{1}^{Z} \leq r_{1}^{Z}-E C_{Z}$.

L'ensemble des accords possibles est:

$$
\begin{aligned}
A=\{ & \left(F_{1}^{Y} ; F_{1}^{Z}\right) \in R^{2}: F_{1}^{Y}+F_{1}^{Z} \geq r_{1}^{X}-1 ; F_{1}^{Y} \leq r_{1}^{Y}-E C_{Y} ; \\
& \left.F_{1}^{Z} \leq r_{1}^{Z}-E C_{Z}\right\}
\end{aligned}
$$

L'ensemble des équilibres de Nash symétriques est:

$$
\begin{aligned}
N=\{ & \left(F_{1}^{Y} ; F_{1}^{Z}\right) \in R^{2}: F_{1}^{Y}+F_{1}^{Z}=r_{1}^{X}-1 ; F_{1}^{Y} \leq r_{1}^{Y}-E C_{Y} ; \\
& \left.F_{1}^{Z} \leq r_{1}^{Z}-E C_{Z}\right\}
\end{aligned}
$$

L'arbitre résout:

$$
\begin{array}{cl}
\text { Max }_{1}^{Y} ; F_{1}^{Z} & {\left[U_{Y}\left(r_{1}^{Y}-F_{1}^{Y}\right)-U_{Y}\left(E C_{Y}\right)\right]\left[U_{Z}\left(r_{1}^{Z}-F_{1}^{Z}\right)-U_{Z}\left(E C_{Z}\right)\right]} \\
\text { sous } & U_{Y}\left(r_{1}^{Y}-F_{1}^{Y}\right) \geq U_{Y}\left(E C_{Y}\right) \\
& U_{Z}\left(r_{1}^{Z}-F_{1}^{Z}\right) \geq U_{Z}\left(E C_{Z}\right) \\
& F_{1}^{Y}+F_{1}^{Z}=r_{1}^{X}-1
\end{array}
$$

La solution de Nash est donnée par:

$$
\frac{U_{Y}^{\prime}\left(r_{1}^{Y}-F_{1}^{Y}\right)}{U_{Y}\left(r_{1}^{Y}-F_{1}^{Y}\right)-U_{Y}\left(E C_{Y}\right)}=\frac{U_{Z}^{\prime}\left(r_{1}^{Z}-r_{1}^{X}+1+F_{1}^{Y}\right)}{U_{Z}\left(r_{1}^{Z}-r_{1}^{X}+1+F_{1}^{Y}\right)-U_{Z}\left(E C_{Z}\right)}
$$

On peut de même facilement traiter les cas où le renflouement est effectué par les agents de type 2 et où il est effectué par les deux types d'agents.

Dans son rôle de gestionnaire de la crise, le PDR (la chambre de compensation ou la Banque centrale) garantit aux déposants de chaque banque que s'ils paniquent, ils recevront le rendement promis avec certitude. Ou en d'autres termes, il crée de la liquidité sans détenir de capital et sans accorder de crédits. Cela lui est possible en garantissant que dans le jeu de négociation des banques solidaires, parmi la multiplicité des équilibres de Nash elles se coordonneront sur un équilibre efficace et équitable. Les déposants n'ont donc aucune incitation à paniquer et la solidarité de place ne sera jamais effectivement mise en oeuvre. 


\section{Les paniques bancaires de 1863 à 1933 aux Etats-Unis et le sauvetage de LTCM}

Notre modèle donne une illustration théorique de la thèse développée par Wicker (2000) sur les paniques bancaires de la période du National Banking aux Etats-Unis. Nous pouvons résumer cette thèse ainsi.

La CCNY n'était pas seulement une institution organisant la collecte et la compensation des chèques. En tant qu'association de banquiers, elle pouvait organiser leur coopération en période de crise bancaire. Deux instruments étaient en sa possession pour gérer les crises: l'émission de certificats de prêts et la mise en commun des réserves.

Les certificats de prêt étaient émis en contrepartie d'un dépôt de titres en garantie. Ces prêts étaient accordés pour effectuer les règlements à la CCNY. Ils circulaient uniquement entre banques. Ce n'étaient pas des espèces circulant dans le public. La monnaie qui n'était pas utilisée pour les règlements interbancaires était disponible pour rembourser les déposants et accorder des crédits. ${ }^{5}$

Dans la mise en commun des réserves, la CCNY prenait le contrôle des réserves des banques membres et transférait les fonds des banques en excédent aux banques en déficit afin de prévenir la suspension de la convertibilité.

Elle avait également le pouvoir d'examiner les comptes des banques subissant des ruées et de certifier leur solvabilité. Des membres de l'association pouvaient aider des banques en difficulté. Les pertes qu'ils pouvaient subir dans ces opérations de soutien étaient couvertes par la CCNY. Les réserves du pays étaient logées dans les plus grandes banques de New York. Selon Sprague (1910), le volume de ces réserves était supérieur à celles détenues par n'importe quelle Banque centrale européenne. Même si la CCNY ne pouvait pas augmenter le volume des réserves, elle avait néanmoins les instruments et les pouvoirs nécessaires pour éviter les paniques. La cause principale des paniques bancaires a été l'incapacité de la CCNY à organiser la coopération.

De 1860 à 1873, la CCNY a eu en la personne de George Simmons Coe un président ayant une forte autorité. Coe était conscient de la responsabilité de la CCNY dans la gestion des paniques. Il fut le concepteur des certificats de prêts et de la mise en commun des réserves.

\footnotetext{
${ }^{5}$ voir Cannon (1910) pour une description plus approfondie
} 
Durant la panique de 1873, la CCNY a autorisé l'émission de certificats de prêts et la mise en commun des réserves. La convertibilité fut néanmoins suspendue quelques jours après. Sprague (1910) explique l'incapacité de la CCNY a éviter les suspensions par le fait que les banques de New York détenaient des réserves insuffisantes avant la panique. Wicker (2000) rejette cette thèse. Il souligne qu'après la suspension les banques de New York ont continué à transférer des réserves aux banques de l'intérieur du pays aussi librement qu'avant la suspension. Cela a été rendu possible par la mise en commun des réserves. En outre, la suspension de la convertibilité s'accompagnait toujours d'une thésaurisation plus importante, ce qui limitait sa capacité à préserver les réserves des banques. La suspension n'était tout simplement pas nécessaire.

En 1884 et 1890, les difficultés bancaires n'ont pas dégénéré en panique grâce à l'intervention rapide de la CCNY. Le 14 mai 1884, à la suite d'une ruée déclenchée par des rumeurs de spéculation sur les titres des compagnies de chemins de fer, la Metropolitan Bank ferme ses portes. Les deux tiers de ses dépôts provenaient d'autres banques. Un effet-domino était donc à craindre. L'après-midi du 14 mai, la CCNY autorise l'émission de certificats de prêts. Les comptes de la banque furent inspectés et elle fut certifiée solvable. La Metropolitan Bank réouvrit ses portes le 15 mai. Le krach boursier et la chute du marché du pétrole entrainèrent quelques faillites bancaires qui n'eurent pas d'effet de contagion. Le 11 novembre 1890, la faillite du courtier Decker, Howell and Co déclenche une panique boursière. Son banquier, la Bank of North America, était menacé de faillite imminente. Le même jour, les membres de la CCNY se réunirent et décidèrent d'assister Bank of North America et Mechanics and Trader's Bank. Avant cette réunion, neuf banques de New York, sous le leaderschip de J.P. Morgan, s'accordèrent pour contribuer à hauteur de $100000 \$$ chacune au renflouement des banques en difficulté. Il n'y a eu aucun effet-domino.

La panique de 1893 est particulière dans l'histoire financière des EtatsUnis. Les ruées des déposants ont touché les banques de l'intérieur du pays mais pas celles de New York. La CCNY n'est pas intervenue pour éviter la panique car les banques de New York n'étaient pas menacées. Elles n'avaient aucune incitation à prévenir la panique.

En 1907, il n'y avait pas de leadership fort au sein de la CCNY. Sa responsabilité dans la prévention des paniques bancaires qui avait été bien comprise par ses membres jusqu'en 1890, avait disparu de leurs préoccupations. 
La panique a touché les "trust companies" qui n'étaient pas membres de la CCNY. En outre, les "trust compnies" n'avaient aucune organisation formelle ou informelle pour faire face aux crises. Lorsque les déposants se sont rués sur Knickerbocker Trust, la CCNY n'est pas intervenue et il s'en est suivi la faillite. La contagion menaçait alors Trust Company of America et Lincoln Trust. Afin d'éviter l'effet-domino, JP Morgan (qui n'était pas membre de la CCNY) organise la coopération d'un groupe de trusts pour sauver celles qui étaient solvables. Mais l'initiative de JP Morgan ne mit pas fin aux ruées qui continuèrent pendant deux semaines, car il était moins crédible que la CCNY. Sprague (1910), Wicker (2000), et Moen et Tallman $(1995,1999,2000)$ partagent la thèse que la cause principale de la panique de 1907 a été que la CCNY n'a pas joué son rôle de PDR car les ruées touchaient les "trust companies" qui n'étaient pas membres de la CCNY.

Wicker (2000) soutient que lorsqu'une autorité suffisamment forte, qui émergeait par hasard, imposait à la CCNY de préserver la stabilité du système bancaire, elle est intervenue pour enrayer les paniques. En revanche, lorsqu'une telle autorité a fait défaut, il n'y a pas eu d'intervention de stabilisation. Ceci a été la principale cause des paniques de 1893 et 1907. Nous retrouvons exactement le même résultat dans notre modèle: la solidarité de place ne peut être effective que s'il existe un arbitre indépendant qui impose la solution de Nash dans le jeu de négociation des banques solidaires. En l'absence d'un tel arbitre, seule une Banque centrale ayant le pouvoir de création monétaire peut éviter les paniques.

Nous n'avons pas développé un modèle de chambre de compensation comme l'ont fait McAndrews et Roberds (1995). L'intérêt de notre modèle est qu'il est simple et permet néanmoins de retrouver deux aspects essentiels des paniques du National Banking selon Wicker (2000): les ruées sur les banques solvables et le problème de leadership.

Wicker (1996) montre comment la simple annonce d'une coopération entre les banques a suffi à calmer la deuxième crise bancaire de 1931. A l'initiative du Président Hoover fut mise en place le 13 octobre la National Credit Corporation. Il s'agissait d'une association de banquiers constituant un pool de crédit de 500 millions de dollars. L'association accorda très peu de prêts. La simple annonce de sa mise en place suffit à calmer la panique. La semaine se terminant le 7 octobre a vu la thésaurisation augmenter de plus de 100 millions de dollars. La semaine suivant l'annonce du Président Hoover, la hausse fut réduite des deux tiers. Nous citons Wicker (1996): 
"The amount of dollar assistance made available in October was minuscule and could not by itself have contributed to alleviating the crisis generated when Britain abandoned gold. Nevertheless, the announcement effects may have played a significant role in reducing the number of bank failures and the amount of hoarding in the week that followed" (page 96).

Wicker cite plus loin Ogden Mills, Sous-secrétaire du Trésor:

"The mere existence of the fund, the mere evidence of the fact that the banking community of the United States stood ready to act with solidarity restored confidence" (page 96). ${ }^{6}$

L'événement qui déclencha la panique bancaire de 1933 fut la déclaration d'un moratoire sur les dépôts le 14 février par le Gouverneur du Michigan. Il faisait suite à l'échec des négociations entre la RFC et les banques du Guardian Group sur le sauvetage de Union Guardian Trust. La panique aurait pu être évitée si une autorité suffisamment forte avait pu imposer une solution ne nécessitant que quelques millions de dollars.

Dans le cas du sauvetage de LTCM, la solidarité de place a effectivement eu lieu, alors que dans notre modèle l'annonce de la solidarité suffit à exclure les paniques. Ceci est bien sûr lié au fait que LTCM avait subi des pertes sur ses investissements. Cet aspect n'apparait pas dans le modèle de DD sans risque d'actif. C'est pourquoi notre modèle ne rend pas compte de façon exhaustive du sauvetage de LTCM. Notre propos est plus modeste. Nous avons simplement tenté de modéliser comment la solidarité de place peut éliminer les crises de liquidité.

Kho, Lee et Stulz (1999) ont étudié l'impact de la crise de LTCM sur les cours des actions des banques. Ils ont trouvé que la débâcle de LTCM n'a pas eu d'effet de contagion dans le système bancaire. Les quatre banques de leur échantillon qui ont participé aux réunions organisées par la Fed de New York (Citicorp, Bankers Trust, JP Morgan, Chase Manhattan) ont perdu 29,45\% de leur capitalisation boursière entre le 26 août et le 4 septembre. Durant cette période, les banques non exposées à LTCM (définies comme celles qui

\footnotetext{
${ }^{6}$ Dans l'affaire du Crédit Suisse de Chiasso, l'annonce par la Banque Nationale de la mise à disposition par l'UBS et la SBS de 3 milliards de francs aggrava la panique. Car jusqu'à cette annonce, il n'était question que d'une perte de l'ordre de 250 millions de francs. Alors pourquoi offrir 3 milliards ? se demanda le public.
} 
n'ont pas participé aux réunions organisées par la Fed de New York) n'ont perdu que 9,35\% de leur capitalisation. Le marché a distingué les banques exposées des banques non exposées. Kho, Lee et Stulz soulignent que trois explications de l'absence de contagion sont envisageables. Premièrement, le risque systémique était effectivement faible. Deuxièmement, le marché a sous-estimé ce risque. Troisièmement, le marché avait confiance dans la capacité des autorités de tutelle à contrôler la situation.

Le cadre théorique que nous avons développé explique simplement, dans le cas d'une crise de liquidité, comment d'une part la faillite du marché monétaire rend nécessaire le transfert interbancaire de capital (ie la solidarité de place). Comment d'autre part la confiance en la capacité des autorités à organiser la solidarité de place élimine les paniques bancaires.

\section{Conclusion}

Dans le modèle de DD, le PDR doit être doté de suffisamment de richesse pour éliminer les crises de liquidité.

Lorsque la ruée sur une banque crée des anticipations de ruées sur d'autres banques, la solidarité de place est une alternative à l'octroi de crédits. Les banques solidaires doivent se répartir la perte sociale générée par la ruée initiale. Ce jeu de négociation possède une infinité d'équilibres de Nash. La solution de Nash est un équilibre focal qui peut être sélectionné par un arbitre indépendant. Les banques solidaires peuvent alors créer de la liquidité sans créer de monnaie

\section{Bibliographie.}

Calomiris Charles, Joseph Mason (2000): Causes of US Bank Distress During The Great Depression, NBER Working Paper, n 7919

Cannon James Graham (1910): Clearing Houses, National Monetary Commission, Document n491. Washington: Government Printing Office.

Carlson Mark (2002): Causes of Bank Suspensions in the Panic of 1893, Finance and Economics Discussion Paper, 2002-11 
Cooper Russell, Thomas Ross (1998): Bank Runs: Deposit Insurance and Capital Requirements, miméo

de Boyer Jérôme (1998): Endoghenous money and shareholder's funds in the classical theory of banking, The European Journal of the History of Economic Thought, Spring, 60-84

de Weck Roger, Max Mabillard (1977): Scandale au Crédit Suisse, Tribune Editions

Diamond Douglas, Philip Dybvig (1983): Bank Runs, Deposit Insurance, and Liquidity, Journal of Political Economy, vol 91, n³, 401-419

Donaldson Glen (1992): Sources of Panics, Evidence from the Weekley data, Journal of Monetary Economics, vol 29, 277-305

Dowd Kevin (2000): Bank Capital Adequacy versus Deposit Insurance, Journal of Financial Services Research, vol 17, n 1, 7-15

Edwards Franklin (1999): Hedge Funds and the Collapse of Long-Term Capital Management, Journal of Economic Perspectives, vol 13, n² 2, 189-210

Engineer Merwan (1989): Bank Runs and the Suspension of Deposit Convertibility, Journal of Monetary Economics, vol 24, 443-454

Fischer Stanley (1999): On the Need for an International Lender of Last Resort, mimeo

Freixas Xavier (1999) The Lender of Last Resort in Today Financial Environment, ELS Opuscles del Centro de Recerca En Economia Internacional, $n^{\circ} 4$

Freixas Xavier, Curzio Giannini, Glenn Hoggarth, Farouk Soussa (2000): Lender of Last Resort: What Have We Learned Since Bagehot ?, Journal of Financial Services Research, vol 18, n¹,63-84

Friedman Milton, Anna Schwartz (1963): A Monetary History of the United States, 1867-1960. Princeton University Press 
Friedman Milton, Anna Schwartz (1986): The Failure of the Bank of United States: A Reappraisal, Explorations in Economic History, 22, 199204

Goodhart Charles, Dirk Schoenmaker (1995): Should The Functions of Monetary Policy And Banking Supervision Be Separated ?, Oxford Economic Papers, vol 47, 539-560

Humphrey Thomas (1975): The Classical Concept of the Lender of Last Resort, Federal Reserve Bank of Richmond Economic Review, january / february, 2-9

Humphrey Thomas (1989): Lender of Last Resort: The Concept in History, Federal Reserve Bank of Richmond Economic Review, march / april

Kho Bong-Chan, Dong Lee, René Stulz (1999): US Banks, Crises, and Bailouts: From Mexico to LTCM, miméo

Lucia Joseph (1985): The Failure of the Bank of United States: A Reappraisal, Explorations in Economic History, 22, 402-416

Madiès Philippe (2000): Les Fondements des Systèmes de Garantie des dépôts et la Prévention des Paniques Bancaires: Analyses Microéconomiques et Etudes expérimentales, Thèse de Doctorat

Marini François (2001): Monnaie, Banque et Capital, Revue d'Economie Politique, à paraitre

McAndrews James, William Roberds (1995): Banks, Payments and Coordination, Journal of Financial Intermediation, $n^{\circ} 4,305-327$

Mc Culloch Huston, Min-Teh Yu (1998): Government Deposit Insurance and the Diamond-Dybvig Model, The Geneva Papers on Risk and Insurance Theory, vol 23, 139-149

Moen Jon, Ellis Tallman (1995): Private Sector Responses to the Panic of 1907: A Comparison of New York and Chicago, Federal Reserve Bank of Atlanta Economic Review, vol 80, n², 1-9 
Moen Jon, Ellis Tallman (1999): Why Didn't the United States Establish a Central Bank until after the Panic of 1907 ?, Federal Reserve Bank of Atlanta Working Paper 99-16

Moen Jon, Ellis Tallman (2000): Clearinghouse Membership and Deposit Contraction during the Panic of 1907, The Journal of Economic History, vol $60, \mathrm{n}^{\circ} 1,145-163$

Myerson Roger (1991): Game Theory, Harvard University Press

Nakaso Hiroshi (2001): The financial Crisis in Japan during the 1990's: how the Bank of Japan responded and the lessons learnt, BIS Papers, $n^{\circ} 6$

O Grada Cormac, Eugene White (2002): Who Panics During Panics ? Evidence From A Nineteenth Century Savings Bank, NBER Working Paper, $\mathrm{n}^{\circ} 8856$

Osborne Martin, Ariel Rubinstein (1991): Bargaining and Markets, Academic Press

Sachs Jeffrey (1998): Creditor Panics: Causes and Remedies, Deutsche Bank Research Note 98-4

Smith Bruce (1991): Bank Panics, Suspensions and Geography: Some Notes on the Contagion of Fear in Banking, Economic Inquiry, April, 230248

Sprague O.M.W (1910): History of Crises Under The National Banking Era, National Monetary Commission. Washington D.C: Government Printing Office

Thomson William (1994): Cooperative Models of Bargaining, Rochester Center for Economic Research Working Paper, $n^{\circ} 380$

Thornton Henry (1802): An Enquiry into the Nature and the Effects of the Paper Credit of Great Britain, London:F.A Hayek, (1839)

van Damme Eric (1991): Stability and Perfection of Nash equilibria, Springer-Verlag 
Wicker Elmus (1996): The Panics of the Great Depression, Cambridge University Press

Wicker Elmus (2000): Banking Panics of the Gilded Age, Cambridge University Press 